\author{
Peter Heusser
}

\title{
Spiritualität in der medizinischen Anthropologie. Warum? Und wie?*
}

Aspekte des Menschseins und damit auch der Bedürfnisse von Patienten.

Mit einer gewissen Verzögerung ist aber auch in der konventionellen Hochschulmedizin das Interesse an komplementärmedizinischen Verfahren und Spiritualität erwacht, und daraus hat sich in verhältnismässig kurzer Zeit eine stark wachsende Forschungsaktivität entfaltet. Diese beschäftigt sich im Bereich Spiritualität vorerst mit Themen wie Prävalenz spiritueller Bedürfnisse und Haltungen, Spiritualität als Ressource bei chronischen Krankheiten oder Einfluss von Spiritualität auf Lebensqualität, Krankheitsverarbeitung und Krankheitsverlauf. Im Rahmen solcher Arbeiten hat auch die direkte Integration komplementärmedizinischer und spiritueller Praktiken in Bereiche der konventionellen Medizin begonnen. In den USA z.B. sind an fast allen führenden Universitäten «Centers for Integrative Medicine» entstanden, die durch öffentliche Gelder und grosse Stiftungen finanziert werden und sich zum «Consortium of Academic Health Centers for Integrative Medicine» zusammengeschlossen haben.

Die medizinische Anthropologie und damit das medizinische Denken über den Menschen ist jedoch von diesen verhältnismässig jungen Entwicklungen vorerst noch unberührt geblieben. So werden im aktuellen medizinischen Menschenbild organische Prozesse nach wie vor auf rein physisch-chemische Prozesse zurückgeführt, die Fragen nach dem Wesen des Lebens und nach dem Leib-SeeleVerhältnis werden meist zugunsten

naturalistischer Erklärungen ent- schieden, das Seelisch-Geistige wird nicht als eigenständige Realität anerkannt und ein Leben nach dem Tod wird oft für eine abgetane hypothetische Spekulation gehalten.

Gleichzeitig beginnt man aber, im Rahmen der integrativmedizinischen Bemühungen die immateriellen Konzepte anderer Kulturen zu rezipieren und in der Praxis anzuwenden. So entsteht die paradoxe Situation, dass sich z.B. in der stark wachsenden integrativen Medizin der USA, wo die Chinesische Medizin überall mächtig Einzug gehalten hat, die Menschenbilder des naturalistischen Westens und des spiritualistischen Ostens ohne konzeptionelle Vermittlung gegenüberstehen. In fast naiv anmutender Weise werden physiologische und pathologische Prozesse einerseits mechanistisch-materialistisch und andererseits spiritualistisch gedeutet, zum Teil von denselben Personen. Dazwischen bleibt ein Abgrund bestehen, der konzeptuell vorerst nicht überbrückbar erscheint, und Versuche, diese Kluft zu überbrücken, sind kaum vorhanden. Denn Wissenschaftlichkeit und Erkennbarkeit wird im Sinne von tradierten Denkgewohnheiten meist nur dem rein Naturwissenschaftlichen attestiert, und die energetischen Konzepte aus spirituellen Kulturen werden auf die Felder der «Philosophie» oder des «Glaubens» verwiesen. So bleibt die für das Mittelalter charakteristische Kluft zwischen «Wissen» und «Glauben» zunächst bestehen. Trotz zunehmender faktischer und studienmässiger Integration komplementärmedizinischer und spiritueller Methoden in die konventionelle Medizin fehlen so
*Deutsche Zusammenfassung des Vortrags «Spirituality in Medical Anthropology. Why? And How?» am II. European Congress (c) 2010 S. Karger GmbH, Freiburg
Prof. Dr. med. Peter Heusser, MME (UniBe)

Lehrstuhl für Medizintheorie, Integrative und Anthroposophische Medizin

Fakultät für Gesundheit, Private Universität Witten/Herdecke gGmbH

Alfred-Herrhausen-Strasse 50, 58448 Witten, Deutschland

peter.heusser@uni-wh.de 
wissenschaftliche Konzepte, die eine gegenseitige Verständigung und eine Integration von Spiritualität und $\mathrm{Na}$ turwissenschaft auch im Bereich der medizinischen Anthropologie erlauben würden.

In der europäischen Geistes- und Wissenschaftsgeschichte sind aber die Grundlagen einer wissenschaftlichen Begründung von Spiritualität vorhanden. So lässt sich etwa anhand der erkenntnis- und wissenschaftsphilosophischen Prinzipien von Plato, Aristoteles, Thomas von Aquin, Albertus Magnus, René Descartes, Johann Gottlieb Fichte, Immanuel Hermann Fichte, Friedrich W.J. Schelling, Georg F.W. Hegel, Johann Wolfgang von Goethe, Rudolf Steiner, Franz Brentano, Nicolai Hartmann, Werner Heisenberg, Carl Friedrich von Weizsäcker, Hans Primas, Vittorio Hösle, Dieter Wandschneider und anderen zeigen, dass das Geistige ein epistemologisch und ontologisch begründbares Fak- tum darstellt, das nicht nur als transzendent erschliessbar, sondern als den physischen und psychischen Phänomenbereichen immanent der empirischen Forschung grundsätzlich zugänglich ist. Auf dieser Grundlage ergeben sich Konzepte der Materie, des Lebens, des Seelischen und des Geistigen, die mit den Fakten der modernen Naturwissenschaft und Psychologie kompatibel sind (ohne jedoch mit den Modellvorstellungen des materialistischen Reduktionismus übereinstimmen zu müssen) und die zudem erlauben, die wissenschaftlich notwendigen Brücken zu bauen zu den ganzheitlichen Konzepten und immateriellen Wirkfaktoren, die von komplementärmedizinischen Methoden und humanistischen Auffassungen des Menschseins geltend gemacht werden.

Daraus lässt sich dann eine medizinische Anthropologie unter Einschluss von Spiritualität entwickeln, die sowohl der berechtigten Forde- rung der modernen Medizin nach empirisch begründbarer Wissenschaftlichkeit wie auch den ebenso berechtigten Bedürfnissen der Bevölkerung nach einer umfassenderen Humanität in der medizinischen Versorgung entgegenkommt und die zudem der geistigen Entwicklung des Abendlandes entspricht, wie sie durch die Ausbreitung des Christentums und die Entfaltung des wissenschaftlichen Bewusstseins seit Aristoteles entstanden ist. Daraus erhellt aber auch, dass dem Medizinstudium eine umfassendere erkenntniswissenschaftliche, philosophische und humanistische Bildung zugrunde gelegt werden müsste, wenn das spirituelle Vakuum behoben werden soll, das durch die einseitige Abstützung der Medizin auf Naturwissenschaft sowie durch den Glauben entstanden ist, Wissenschaft sei auf Naturwissenschaft und Spiritualität auf den Glauben zu begrenzen. 